\title{
Gut Microbiota as a Driver of Inflammation in Nonalcoholic Fatty Liver Disease
}

\author{
Stefano Bibbò $\mathbb{D}^{1},{ }^{1}$ Gianluca Ianiro, ${ }^{2}$ Maria Pina Dore $\left(\mathbb{D},{ }^{1}\right.$ Claudia Simonelli, ${ }^{2}$ \\ Estelle E. Newton, ${ }^{3}$ and Giovanni Cammarota ${ }^{2}{ }^{2}$ \\ ${ }^{1}$ Department of Clinical and Experimental Medicine, University of Sassari, Sassari, Italy \\ ${ }^{2}$ Department of Gastroenterology, Catholic University, School of Medicine and Surgery, A. Gemelli Hospital, Rome, Italy \\ ${ }^{3}$ CytoCure LLC, Beverly, MA, USA \\ Correspondence should be addressed to Giovanni Cammarota; Giovanni.Cammarota@unicatt.it
}

Received 5 May 2017; Revised 12 July 2017; Accepted 26 July 2017; Published 31 January 2018

Academic Editor: Jorg Fritz

Copyright ( 2018 Stefano Bibbò et al. This is an open access article distributed under the Creative Commons Attribution License, which permits unrestricted use, distribution, and reproduction in any medium, provided the original work is properly cited.

\begin{abstract}
The prevalence of nonalcoholic fatty liver disease and the consequent burden of metabolic syndrome have increased in recent years. Although the pathogenesis of nonalcoholic fatty liver disease is not completely understood, it is thought to be the hepatic manifestation of the dysregulation of insulin-dependent pathways leading to insulin resistance and adipose tissue accumulation in the liver. Recently, the gut-liver axis has been proposed as a key player in the pathogenesis of NAFLD, as the passage of bacteria-derived products into the portal circulation could lead to a trigger of innate immunity, which in turn leads to liver inflammation. Additionally, higher prevalence of intestinal dysbiosis, larger production of endogenous ethanol, and higher prevalence of increased intestinal permeability and bacterial translocation were found in patients with liver injury. In this review, we describe the role of intestinal dysbiosis in the activation of the inflammatory cascade in NAFLD.
\end{abstract}

\section{Introduction}

Nonalcoholic fatty liver disease (NAFLD) is a multifactorial condition resulting from a complex interaction of genetic and environmental factors. The prevalence of the disorder has increased in recent decades, as is the burden of metabolic syndrome [1]. NAFLD was defined as a spectrum of liver conditions whose dominant feature is abnormal hepatic triglyceride accumulation. In the absence of inflammation and hepatocellular damage, this condition is simply defined as steatosis or nonalcoholic fatty liver. In a liver with chronic NAFLD, lobular inflammation and signs of hepatocellular damage may occur. This latter condition is called nonalcoholic steatohepatitis (NASH). The natural history of NASH is not completely understood, but one can assume that NASH predisposes to several complications such as liver fibrosis, cirrhosis, and hepatocellular carcinoma [2,3]. Moreover, the pathogenesis of this disorder remains largely unknown. The so-called two-hit hypothesis suggests that accumulation of triglyceride in hepatic cells may expose the liver to secondary insults, primarily oxidative stress, resulting in chronic injury. This model focuses on liver autonomous dysfunction leading to NASH [4]. In recent years, NAFLD was proposed as the hepatic feature of metabolic disorders, as insulin resistance and metabolic syndrome are strongly linked to the progression of liver disease. However, other organs including adipose tissue, muscle, and gut may play an important role in the progression of NAFLD $[1,3]$. The liver and intestine are tightly linked through the portal circulation; consequently, gut-derived products, mainly microbial components, arrive primarily to the liver with obvious pathogenic implications [5]. The intestine is colonized by an enormous array of microorganisms, defined as the gut microbiota or microbiome, which can be considered a functional organ [6]. The gut microbiota plays a key role in the maintenance of human health, being involved in the development and growth of the immune system and regulation of several metabolic pathways [7-9]. Quantitative and/or qualitative alterations of gut microbiota, in other way defined as dysbiosis, are known to lead to disruption of this 
homeostasis and, consequently, development of pathology. Disorders associated with the impairment of gut microbiota can include gastrointestinal diseases [10-13], liver diseases [14], and also metabolic disorders such as metabolic syndrome [15] and diabetes [16, 17].

\section{The Role of Intestinal Dysbiosis}

Our understanding of the relationship between gut microbiota and the development of liver disease has been highlighted in both animal and human studies (see Table 1). Small intestinal bacterial overgrowth (SIBO), increased intestinal permeability, and a number of bacterial endotoxins were reported as putative factors for NASH development [18]. In the first observation, the authors hypothesized an important role for SIBO in the occurrence of NASH, but in recent years, the development of metagenomic sequencing technologies has allowed the description of detailed alterations of the gut microbiota, focusing on qualitative dysbiosis rather than quantitative modifications [19-22].

The prevalence of SIBO in patients with NASH has been widely studied. Wigg et al. reported that patients with NASH have a higher prevalence of SIBO compared to controls $(50 \%$ versus $22 \%$ ). In addition, higher levels of TNF-alpha compared to control subjects were observed, although intestinal permeability and serum endotoxin levels were similar in the two groups [23]. These findings were only partially confirmed by further studies. Miele et al. found that subjects with NAFLD had significantly increased gut permeability and a higher prevalence of SIBO, compared with healthy subjects. Both gut permeability and the prevalence of SIBO correlated with the severity of steatosis but not with the presence of NASH [24]. These findings were confirmed in a further study by Shanab et al.; additionally, authors found an enhanced expression of Toll-like receptor 4 (TLR4) and release of interleukin 8 (IL-8) [25]. Moreover, increased intestinal permeability and higher levels of blood lipopolysaccharide (LPS) were found in children with NASH compared to those with NAFLD [26]. These data confirmed findings based on rodent models, showing that higher intestinal mucosa permeability promotes the increase of LPS levels in portal blood and in turn liver inflammatory damage [27-29].

The gut microbiota has been suggested to be responsible for the increase of endogenous ethanol production in patients with NAFLD. A rodent experimental model demonstrated an increased breath ethanol content [30] that was abolished by treatment with neomycin. This observation was also confirmed in humans. Patients with NASH harbored an increased number of alcohol-producing bacteria (in particular Escherichia coli) in their microbiome associated with elevated blood-ethanol concentration [31]. An additional study confirmed the results [32]. Patients with NAFLD had a prevalence of SIBO of $37.5 \%$, and Escherichia coli was the predominant bacteria in duodenal fluid aspirate. Moreover, patients with SIBO had higher endotoxin levels and expression of Toll-like receptor 4 (TLR4) compared to those without [32]. However, the presence of SIBO appears not to represent an ubiquitous marker of NAFLD. A study performed on 20 patients with NAFLD showed intestinal permeability, and alcohol and endotoxin levels in the plasma were significantly higher compared to controls, but the prevalence of SIBO was similar between patients and controls [33].

The development of modern sequencing techniques (metagenomic approach) has allowed a deeper analysis of the microbiota composition [34]. The first metagenomic characterization of gut microbiota in patients with NASH was reported by Mouzaki et al. [35]. The percentage of Bacteroidetes and C. coccoides was lower in patients with NASH compared to patients with NAFLD and healthy controls. The percentage of Bacteroidetes in patients with NASH remained significantly lower even after adjusting for anthropometric variables (body mass index) and fat intake [35]. Boursier et al. were able to partially confirm the data. In their study, patients with NASH harbored a higher quantity of Bacteroides and a lower quantity of Prevotella, compared to individuals without NASH [36]. The multivariate analysis adjusted for metabolic factors showed that Bacteroides abundance was independently associated with NASH. Differences in taxonomic composition of intestinal microbiota at the phylum level according to NAFLD severity were not detected. On the contrary, dramatic differences were observed at the family level according to severity of hepatic injury. More specifically, Bacteroidaceae family increased along with severity of liver lesions, whereas the family of Prevotellaceae and Erysipelotrichaceae decreased. Authors also evaluated the correlation with the grade of liver fibrosis. Patients with a grade of liver fibrosis of F0/F1 had higher abundances of Bacteroides and Ruminococcus and lower abundance of Prevotella compared to those with F2 liver fibrosis [36]. Analysis of the fecal microbiome and volatile organic compound (for instance ethanol) in patients with $\mathrm{NASH}$ revealed a significant increase in fecal volatile compounds in NAFLD patients compared to healthy controls. In the microbiome of NAFLD patients, Lactobacillus species and selected members of phylum Firmicutes, in particular Lachnospiraceae (Dorea, Robinsoniella, and Roseburia), were overrepresented, while other members (Ruminococcaceae; genus, Oscillibacter) were significantly underrepresented [37]. Further data show that patients with NASH have a higher abundance of Parabacteroides and Allisonella and lower representation of Faecalibacterium and Firmicutes families [38].

The intestinal dysbiosis is able to modify the profile of bile acids in patients with NAFLD. In a population of patients with NASH, levels of unconjugated cholic acid and chenodeoxycholic acid were, respectively, increased. The analysis of intestinal microbiota revealed that patients with NASH harbored a lower relative abundance of Bacteroidetes and Clostridium leptum, independently from other metabolic factors [39]. For instance, Clostridium leptum is able to modify bile acids, converting them from primary to secondary bile acids [40]. The correlation of bile acid levels and intestinal dysbiosis with markers of hepatic injury suggests a possible role for bile acids in the progression of NAFLD to NASH [39].

In pediatric patients, NAFLD-specific alterations in gut microbiota composition, different from those found in adults, were also described. Children with NASH had an 
TABLE 1: Gut microbiota alteration in human studies (NASH = nonalcoholic steatohepatitis; $\mathrm{SS}=$ simple steatosis; HC = healthy controls).

\begin{tabular}{|c|c|c|}
\hline Study & Subjects & Gut microbiota alterations \\
\hline Mouzaki et al. [35] & NAFLD (SS or NASH) and HC & $\begin{array}{l}\uparrow \text { Clostridium coccoides in NASH versus SS } \\
\downarrow \text { Bacteroidetes in NASH versus SS and HC }\end{array}$ \\
\hline Boursier et al. [36] & $\begin{array}{l}\text { NAFLD (SS, NASH, } \\
\text { and fibrosis) }\end{array}$ & $\begin{array}{c}\uparrow \text { Bacteroides and } \downarrow \text { Prevotella in NASH } \\
\uparrow \text { Bacteroidaceae; } \downarrow \text { Prevotellaceae and Erysipelotrichaceae according to the } \\
\text { severity of NASH } \\
\uparrow \text { Bacteroides and Ruminococcus and } \downarrow \text { Prevotella in patients with } \\
\text { F2 fibrosis versus F0/F1 }\end{array}$ \\
\hline Raman et al. [37] & NAFLD and HC & $\begin{array}{l}\uparrow \text { Lactobacillus and selected members of Firmicutes (Dorea, Robinsoniella, } \\
\text { and Roseburia); } \downarrow \text { one member of Firmicutes (Oscillibacter) in NAFLD }\end{array}$ \\
\hline Wong et al. [38] & $\mathrm{NASH}$ and $\mathrm{HC}$ & $\uparrow$ Parabacteroides and Allisonella; $\downarrow$ Firmicutes and Faecalibacterium in NASH \\
\hline Mouzaki et al. [39] & $\begin{array}{l}\text { NAFLD (SS and NASH) } \\
\text { and HC }\end{array}$ & $\downarrow$ Bacteroidetes and Clostridium leptum in NASH versus HC \\
\hline Zhu et al. [31] & $\begin{array}{l}\text { Children-NASH, obese, } \\
\text { and HC }\end{array}$ & $\begin{array}{c}\uparrow \text { Bacteriodetes and Proteobacteria and } \downarrow \text { Firmicutes and Actinobacteria in } \\
\text { NASH versus HC }\end{array}$ \\
\hline Del Chierico et al. [41] & $\begin{array}{c}\text { Children-NAFLD } \\
\text { (SS and NASH), } \\
\text { obese, and HC }\end{array}$ & $\begin{array}{c}\uparrow \text { Bradyrhizobium, Anaerococcus, Peptoniphilus, Propionibacterium acnes, Dorea, } \\
\text { and Ruminococcus and } \downarrow \text { Oscillospira and Rikenellaceae in NAFLD }\end{array}$ \\
\hline
\end{tabular}

increased number of Bacteriodetes and Proteobacteria and a decreased number of Firmicutes and Actinobacteria compared to healthy children [31]. In a more recent research, a similar dysbiosis pattern was observed in pediatric patients characterized by a decrease in Oscillospira and Rikenellaceae and an increase in Bradyrhizobium, Anaerococcus, Peptoniphilus, Propionibacterium acnes, Dorea, and Ruminococcus [41].

\section{The Role of Immunity}

As discussed before, dysbiosis plays a main role in increasing intestinal permeability, with consequent passage into the portal circulation of bacteria-derived products. Among these, the lypopolisaccharide (LPS), a cell component of Gramnegative bacteria, is the best investigated. LPS is able to activate Toll-like receptors (TLRs) resulting in the production of proinflammatory cytokines and chemokines. Several experimental models of NASH reported high levels of LPS leading to hepatic injury through the recruitment of inflammatory cells $[5,42]$. In this pathway, a key role is played by Kupffer cells. They contribute to endotoxin clearance [43] and to inflammatory response, through several TLRs located in their surface that, after being activated by LPS, are able to trigger a cascade of events, leading to the production of inflammatory cytokines, such as IL- $1 \beta$ and TNF- $\alpha$ [44].

Up to 13 different TLRs have been identified in mammals; however, among these, only TLR2, TLR4, TLR5, TLR6, TLR7, and TLR9 are known to be involved in the pathogenesis of NAFLD [45]. TLR2 mainly binds peptidoglycan and lipoteichoic acid that are components of Gram-positive bacterial cells. In a murine experiment, Miura et al. [45] demonstrated that TLR2-deficient mice are resistant to diet-induced steatohepatitis, showing a lower expression of proinflammatory cytokines (TNF $\alpha$ and IL-1 $\beta$ ) [46]. In contrast, in other experiments on TLR2-deficient mice, a similar and even more severe susceptibility to steatohepatitis was observed $[47,48]$. Studies looking at the interaction between TLR2 and TLR6 found that deregulation of TLR6 expression potentiated the TLR2-mediated liver inflammation. Indeed, the TLR2/TLR6 stimulation promoted the production of proinflammatory cytokines that was higher in patients with lobular inflammation [49]. TLR4 is a receptor for LPS. The importance of this axis has been clarified through TLR4 mutant mice resistant to the development of NAFLD [50]; furthermore, a direct link between TLR4 and Kupffer cells was described in the pathogenesis of steatohepatitis, as the experimental destruction of Kupffer cells was shown to prevent the increased expression of TLR4 [51]. The relevance of this interplay was confirmed in a murine model, where occurrence of NAFLD required endotoxin-dependent activation of hepatic Kupffer cells, associated with SIBO and enhanced intestinal permeability [52]. TLR5 is a receptor for bacterial flagellin. Although few data are reported about its role in the development of metabolic disorders, a murine model suggests that TLR5 deficiency is able to promote obesity, steatosis, and in turn metabolic syndrome [53]. More recent evidence shows that hepatocyte TLR5 protects against diet-induced liver disease [54]. Similarly, a protective role in preventing NAFLD was also reported for TLR7 [55]. TLR9 is a receptor for bacterial DNA, in particular for the unmethylated CpG motif, which is increased in NASH models; the activation of TLR9 signaling on Kupffer cells induces the production of proinflammatory cytokine, such as IL- $1 \beta$ leading to steatosis and inflammation. Moreover, the activation of TLR9 in hepatic stellate cells suggests a role in promoting fibrogenesis [56]. In animal models, the blockage of Il-1 signaling leads to a reduction of TLR9-mediated liver damage, in particular the endogenous IL-1 receptor antagonist, and regulates the extent of TLR9-induced liver injury [57].

The myeloid differentiation primary response gene 88 (MyD88) is the most investigated signaling adaptor for TLRs. The activation of this adaptor by TLRs, mainly TLR4 and TLR9, results in the upregulation of the transcriptional factor 




FIGURE 1: Interactions between gut microbiota and innate immunity in the pathogenesis of steatohepatitis.

nuclear factor kappa beta (NF- $\kappa \mathrm{B})$ and c-Jun N-terminal kinase (JNK) pathway [58]. However, data about the role of Myd88 in the pathophysiology of NAFLD are conflicting. For example, Duparc et al. recently reported that, in a rodent model, the hepatocyte specific deletion of Myd88 predisposes to inflammation, hepatic steatosis, and insulin resistance [59]; other reports suggested that the deletion of Myd88 increases the risk of developing features of metabolic syndrome such as diabetes and hepatic steatosis [60, 61]; conversely, deletion of MyD88 in intestinal epithelial cellspecific murine model partially protected against dietinduced obesity, diabetes, and metabolic inflammation [62].

Finally, the inflammasome, a group of sensors for endogenous and/or exogenous pathogen-associated molecular patterns (PAMPs) or damage-associated molecular patterns (DAMPs) [63], seems to be involved in development of liver steatosis and inflammation. The inflammasome is a multimeric signaling platform that leads to the production of IL-18 and IL-1 through NRLP3 (NOD-like receptors, pyrin domain containing 3) and NRLP6 (and 6). Interestingly, in inflammasome-deficient mice, an increase of Bacteroidetes and a reduction of Firmicutes were reported, resulting in a higher activation of TLR4 and TLR9 and subsequent inflammatory pathway [64].

\section{Concluding Remarks}

Gut microbiota alterations and increased intestinal permeability appear to play a major role in promoting inflammation and progression of NAFLD to NASH. The disruption of "normal" microbiota can occur in several conditions including environmental exposures, medications, or diet $[65,66]$. It was hypothesized that intestinal dysbiosis may lead to the progression of NAFLD through several pathways. The presence of SIBO is related to endogenous production of alcohol and furthermore to increased intestinal permeability, favoring the passage of bacterial-derived products in the portal circulation. These products (LPS, peptidoglycan, lipoteichoic acid, flagellin, and bacterial DNA) are ligands for TLRs and stimulate the innate immune system in the liver (Figure 1).

Several TLRs, identified in the liver, have a mandatory role in hepatic injury mechanisms, as reported in some animal studies described specifically in the previous section. It has been described that different bacterial products have a selectivity for TLRs, which have different roles in the progression of tissue inflammation. For example TLR4 and TLR9, which bind LPS and bacterial DNA, respectively, promote inflammation and liver fibrogenesis through the activation of Kuppfer cells and hepatic stellate cells. Conversely, certain receptors for bacterial-derived products may have a protective role in the progression of inflammation; indeed, it has been observed that the specific deletion of TLR5 and TLR7 promotes the inflammatory pathways.

Consequently, the altered balance of these receptors can trigger a cascade of events, in particular the secretion of proinflammatory cytokines that drive the inflammation in NAFLD. This condition, previously described as "metabolic endotoxiemia," is a common feature of several metabolic disorders [28, 67].

The reported evidences about the inflammatory pathways mainly derive from animal models. These findings are sometimes conflicting; furthermore, they are not always confirmed by "human" studies. For example, the hepatic deletion of MyD88 seems to promote a proinflammatory "milieu," while the specific deletion in intestinal epithelial cells may have a protective role. There is still much to be understood about the role of the intestine in the inflammatory mechanisms of NAFLD.

In addition, qualitative alterations of gut microbiota are able to interfere with the intestinal absorption of bile acid. Based on this observation, a new role for gut microbiota was proposed. More specifically, intestinal dysbiosis resulting in higher levels of unconjugated bile acid, able to inhibit farnesoid X receptor (FXR) signaling, was observed in animal models [68]. FXR inhibition results in increased production of ceramides that cause lipid toxicity and increased fatty acid synthesis.

In conclusion, to date, evidence for a role of gut microbiota in the progression of NAFLD is still weak, although the reported observations are very intriguing [69]. Research fields that need to be explored are many, from the identification of specific alterations of the gut microbiota, to a more detailed understanding of the mechanisms of innate immunity. The comprehension of the pathogenic pathways of NAFLD in lean patients is a very interesting issue, and several evidences suggest a main role for gut microbiota [70].

These observations allow us to consider a new role for the intestine, suggesting it as one of the main actors in NAFLD/ NASH progression. Indeed, the altered production of volatile metabolites by gut microbiota, such as endogenous alcohol, and the uncontrolled passage of bacterial-derived products 
in the bloodstream would be able to trigger the inflammation and cellular damage in the liver, even in subjects without overt metabolic syndrome. Assuming these data, the gut can be placed side by side with muscle and adipose tissue as a "director" in the progression of liver disease; it would be very important to understand whether intestinal dysbiosis is a factor necessary for the development of NASH, or is only a precipitating factor.

Finally, further studies are needed, and maybe in a future not too far, they will provide new therapeutic chances for this disorder of growing worldwide interest.

\section{Conflicts of Interest}

The authors declare that there is no conflict of interest regarding the publication of this paper.

\section{References}

[1] The Italian Association for the Study of the Liver (AISF), "AISF position paper on nonalcoholic fatty liver disease (NAFLD): updates and future directions," Digestive and Liver Disease, vol. 49, no. 5, pp. 471-483, 2017.

[2] T. Hardy, F. Oakley, Q. M. Anstee, and C. P. Day, "Nonalcoholic fatty liver disease: pathogenesis and disease spectrum," Annual Review of Pathology: Mechanisms of Disease, vol. 11, no. 1, pp. 451-496, 2016.

[3] J. T. Haas, S. Francque, and B. Staels, "Pathophysiology and mechanisms of nonalcoholic fatty liver disease," Annual Review of Physiology, vol. 78, no. 1, pp. 181-205, 2016.

[4] C. P. Day and O. F. James, "Steatohepatitis: a tale of two "hits"?," Gastroenterology, vol. 114, no. 4, pp. 842-845, 1998.

[5] K. Brandl, V. Kumar, and L. Eckmann, "Gut-liver axis at the frontier of host-microbial interactions," American Journal of Physiology-Gastrointestinal and Liver Physiology, vol. 312, no. 5, pp. G413-G419, 2017.

[6] M. Rajilic-Stojanovic and W. M. de Vos, "The first 1000 cultured species of the human gastrointestinal microbiota," FEMS Microbiology Reviews, vol. 38, no. 5, pp. 996-1047, 2014.

[7] M. Rajilic-Stojanovic, "Function of the microbiota," Best Practice \& Research Clinical Gastroenterology, vol. 27, no. 1, pp. 5-16, 2013.

[8] I. Sekirov, S. L. Russell, L. C. M. Antunes, and B. B. Finlay, "Gut microbiota in health and disease," Physiological Reviews, vol. 90, no. 3, pp. 859-904, 2010.

[9] P. J. Turnbaugh, R. E. Ley, M. Hamady, C. M. Fraser-Liggett, R. Knight, and J. I. Gordon, "The human microbiome project," Nature, vol. 449, no. 7164, pp. 804-810, 2007.

[10] M. Simrén, G. Barbara, H. J. Flint et al., "Intestinal microbiota in functional bowel disorders: a Rome foundation report," Gut, vol. 62, no. 1, pp. 159-176, 2013.

[11] S. Bibbò, L. R. Lopetuso, G. Ianiro, T. Di Rienzo, A. Gasbarrini, and G. Cammarota, "Role of microbiota and innate immunity in recurrent Clostridium difficile infection," Journal of Immunology Research, vol. 2014, Article ID 462740, 8 pages, 2014.

[12] D. Viggiano, G. Ianiro, G. Vanella et al., "Gut barrier in health and disease: focus on childhood," European Review for Medical and Pharmacological Sciences, vol. 19, no. 6, pp. 1077-1085, 2015.

[13] G. Cammarota, G. Ianiro, R. Cianci, S. Bibbò, A. Gasbarrini, and D. Currò, "The involvement of gut microbiota in inflammatory bowel disease pathogenesis: potential for therapy," Pharmacology \& Therapeutics, vol. 149, pp. 191-212, 2015.

[14] B. Schnabl and D. A. Brenner, "Interactions between the intestinal microbiome and liver diseases," Gastroenterology, vol. 146, no. 6, pp. 1513-1524, 2014.

[15] M. Nieuwdorp, P. W. Gilijamse, N. Pai, and L. M. Kaplan, "Role of the microbiome in energy regulation and metabolism," Gastroenterology, vol. 146, no. 6, pp. 1525-1533, 2014.

[16] S. Bibbo, M. P. Dore, G. M. Pes, G. Delitala, and A. P. Delitala, "Is there a role for gut microbiota in type 1 diabetes pathogenesis?," Annals of Medicine, vol. 49, no. 1, pp. 11-22, 2016.

[17] G. Muscogiuri, G. Balercia, L. Barrea et al., "Gut: a key player in the pathogenesis of type 2 diabetes?," Critical Reviews in Food Science and Nutrition, pp. 1-16, 2016.

[18] Z. Mokhtari, D. L. Gibson, and A. Hekmatdoost, "Nonalcoholic fatty liver disease, the gut microbiome, and diet," Advances in Nutrition: An International Review Journal, vol. 8, no. 2, pp. 240-252, 2017.

[19] A. Wieland, D. N. Frank, B. Harnke, and K. Bambha, "Systematic review: microbial dysbiosis and nonalcoholic fatty liver disease," Alimentary Pharmacology \& Therapeutics, vol. 42, no. 9, pp. 1051-1063, 2015.

[20] A. R. Moschen, S. Kaser, and H. Tilg, "Non-alcoholic steatohepatitis: a microbiota-driven disease," Trends in Endocrinology and Metabolism, vol. 24, no. 11, pp. 537-545, 2013.

[21] M. Sanduzzi Zamparelli, D. Compare, P. Coccoli et al., "The metabolic role of gut microbiota in the development of nonalcoholic fatty liver disease and cardiovascular disease," International Journal of Molecular Sciences, vol. 17, no. 8, p. 1225, 2016.

[22] E. M. Quigley, A. Abu-Shanab, E. F. Murphy, C. Stanton, and H. P. Monsour Jr, "The metabolic role of the microbiome: implications for NAFLD and the metabolic syndrome," Seminars in Liver Disease, vol. 36, no. 04, pp. 312-316, 2016.

[23] A. J. Wigg, I. C. Roberts-Thomson, R. B. Dymock, P. J. McCarthy, R. H. Grose, and A. G. Cummins, "The role of small intestinal bacterial overgrowth, intestinal permeability, endotoxaemia, and tumour necrosis factor $\alpha$ in the pathogenesis of non-alcoholic steatohepatitis," Gut, vol. 48, no. 2, pp. 206-211, 2001.

[24] L. Miele, V. Valenza, G. La Torre et al., "Increased intestinal permeability and tight junction alterations in nonalcoholic fatty liver disease," Hepatology, vol. 49, no. 6, pp. 1877-1887, 2009.

[25] A. A. Shanab, P. Scully, O. Crosbie et al., "Small intestinal bacterial overgrowth in nonalcoholic steatohepatitis: association with toll-like receptor 4 expression and plasma levels of interleukin 8," Digestive Diseases and Sciences, vol. 56, no. 5, pp. 1524-1534, 2011.

[26] V. Giorgio, L. Miele, L. Principessa et al., "Intestinal permeability is increased in children with non-alcoholic fatty liver disease, and correlates with liver disease severity," Digestive and Liver Disease, vol. 46, no. 6, pp. 556-560, 2014.

[27] P. Brun, I. Castagliuolo, V. Di Leo et al., "Increased intestinal permeability in obese mice: new evidence in the pathogenesis of nonalcoholic steatohepatitis," American Journal of Physiology-Gastrointestinal and Liver Physiology, vol. 292, no. 2, pp. G518-G525, 2007.

[28] P. D. Cani, J. Amar, M. A. Iglesias et al., "Metabolic endotoxemia initiates obesity and insulin resistance," Diabetes, vol. 56, no. 7, pp. 1761-1772, 2007. 
[29] G. L. Su, "Lipopolysaccharides in liver injury: molecular mechanisms of Kupffer cell activation," American Journal of Physiology-Gastrointestinal and Liver Physiology, vol. 283, no. 2, pp. G256-G265, 2002.

[30] K. Cope, T. Risby, and A. M. Diehl, "Increased gastrointestinal ethanol production in obese mice: implications for fatty liver disease pathogenesis," Gastroenterology, vol. 119, no. 5, pp. 1340-1347, 2000.

[31] L. Zhu, S. S. Baker, C. Gill et al., "Characterization of gut microbiomes in nonalcoholic steatohepatitis (NASH) patients: a connection between endogenous alcohol and NASH," Hepatology, vol. 57, no. 2, pp. 601-609, 2013.

[32] S. Kapil, A. Duseja, B. K. Sharma et al., "Small intestinal bacterial overgrowth and toll-like receptor signaling in patients with non-alcoholic fatty liver disease," Journal of Gastroenterology and Hepatology, vol. 31, no. 1, pp. 213-221, 2016.

[33] V. Volynets, M. A. Küper, S. Strahl et al., "Nutrition, intestinal permeability, and blood ethanol levels are altered in patients with nonalcoholic fatty liver disease (NAFLD)," Digestive Diseases and Sciences, vol. 57, no. 7, pp. 1932-1941, 2012.

[34] J. M. Norman, S. A. Handley, and H. W. Virgin, "Kingdomagnostic metagenomics and the importance of complete characterization of enteric microbial communities," Gastroenterology, vol. 146, no. 6, pp. 1459-1469, 2014.

[35] M. Mouzaki, E. M. Comelli, B. M. Arendt et al., "Intestinal microbiota in patients with nonalcoholic fatty liver disease," Hepatology, vol. 58, no. 1, pp. 120-127, 2013.

[36] J. Boursier, O. Mueller, M. Barret et al., "The severity of nonalcoholic fatty liver disease is associated with gut dysbiosis and shift in the metabolic function of the gut microbiota," Hepatology, vol. 63, no. 3, pp. 764-775, 2016.

[37] M. Raman, I. Ahmed, P. M. Gillevet et al., "Fecal microbiome and volatile organic compound metabolome in obese humans with nonalcoholic fatty liver disease," Clinical Gastroenterology and Hepatology, vol. 11, no. 7, pp. 868-875.e3, 2013.

[38] V. W.-S. Wong, C.-H. Tse, T. T.-Y. Lam et al., "Molecular characterization of the fecal microbiota in patients with nonalcoholic steatohepatitis - a longitudinal study," PLoS One, vol. 8, no. 4, article e62885, 2013.

[39] M. Mouzaki, A. Y. Wang, R. Bandsma et al., "Bile acids and dysbiosis in non-alcoholic fatty liver disease," PLoS One, vol. 11, no. 5, article e0151829, 2016.

[40] E. J. Stellwag and P. B. Hylemon, "7alpha-Dehydroxylation of cholic acid and chenodeoxycholic acid by Clostridium leptum," Journal of Lipid Research, vol. 20, no. 3, pp. 325-333, 1979.

[41] F. Del Chierico, V. Nobili, P. Vernocchi et al., "Gut microbiota profiling of pediatric nonalcoholic fatty liver disease and obese patients unveiled by an integrated meta-omics-based approach," Hepatology, vol. 65, no. 2, pp. 451-464, 2017.

[42] R. F. Schwabe, E. Seki, and D. A. Brenner, "Toll-like receptor signaling in the liver," Gastroenterology, vol. 130, no. 6, pp. 1886-1900, 2006.

[43] E. S. Fox, P. Thomas, and S. A. Broitman, "Clearance of gut-derived endotoxins by the liver. Release and modification of ${ }^{3} \mathrm{H},{ }^{14} \mathrm{C}$-lipopolysaccharide by isolated rat Kupffer cells," Gastroenterology, vol. 96, no. 2, pp. 456-461, 1989.

[44] H. Kudo, T. Takahara, Y. Yata, K. Kawai, W. Zhang, and T. Sugiyama, "Lipopolysaccharide triggered TNF- $\alpha$-induced hepatocyte apoptosis in a murine non-alcoholic steatohepatitis model," Journal of Hepatology, vol. 51, no. 1, pp. 168-175, 2009.
[45] K. Miura and H. Ohnishi, "Role of gut microbiota and toll-like receptors in nonalcoholic fatty liver disease," World Journal of Gastroenterology, vol. 20, no. 23, pp. 7381-7391, 2014.

[46] K. Miura, L. Yang, N. van Rooijen, D. A. Brenner, H. Ohnishi, and E. Seki, "Toll-like receptor 2 and palmitic acid cooperatively contribute to the development of nonalcoholic steatohepatitis through inflammasome activation in mice," Hepatology, vol. 57, no. 2, pp. 577-589, 2013.

[47] G. Szabo, A. Velayudham, L. Romics Jr., and P. Mandrekar, "Modulation of non-alcoholic steatohepatitis by pattern recognition receptors in mice: the role of toll-like receptors 2 and 4," Alcoholism: Clinical \& Experimental Research, vol. 29, Supplement, pp. 140S-145S, 2005.

[48] C. A. Rivera, L. Gaskin, M. Allman et al., "Toll-like receptor-2 deficiency enhances non-alcoholic steatohepatitis," BMC Gastroenterology, vol. 10, no. 1, p. 52, 2010.

[49] M. Arias-Loste, P. Iruzubieta, Á. Puente et al., "Increased expression profile and functionality of TLR6 in peripheral blood mononuclear cells and hepatocytes of morbidly obese patients with non-alcoholic fatty liver disease," International Journal of Molecular Sciences, vol. 17, no. 11, article 1878, 2016.

[50] T. Csak, A. Velayudham, I. Hritz et al., "Deficiency in myeloid differentiation factor-2 and toll-like receptor 4 expression attenuates nonalcoholic steatohepatitis and fibrosis in mice," American Journal of Physiology-Gastrointestinal and Liver Physiology, vol. 300, no. 3, pp. G433-G441, 2011.

[51] C. A. Rivera, P. Adegboyega, N. van Rooijen, A. Tagalicud, M. Allman, and M. Wallace, "Toll-like receptor-4 signaling and Kupffer cells play pivotal roles in the pathogenesis of non-alcoholic steatohepatitis," Journal of Hepatology, vol. 47, no. 4, pp. 571-579, 2007.

[52] A. Spruss, G. Kanuri, S. Wagnerberger, S. Haub, S. C. Bischoff, and I. Bergheim, "Toll-like receptor 4 is involved in the development of fructose-induced hepatic steatosis in mice," Hepatology, vol. 50, no. 4, pp. 1094-1104, 2009.

[53] M. Vijay-Kumar, J. D. Aitken, F. A. Carvalho et al., "Metabolic syndrome and altered gut microbiota in mice lacking toll-like receptor 5," Science, vol. 328, no. 5975, pp. 228-231, 2010.

[54] L. Etienne-Mesmin, M. Vijay-Kumar, A. T. Gewirtz, and B. Chassaing, "Hepatocyte toll-like receptor 5 promotes bacterial clearance and protects mice against high-fat dietinduced liver disease," Cellular and Molecular Gastroenterology and Hepatology, vol. 2, no. 5, pp. 584-604, 2016.

[55] S. Kim, S. Park, B. Kim, and J. Kwon, "Toll-like receptor 7 affects the pathogenesis of non-alcoholic fatty liver disease," Scientific Reports, vol. 6, no. 1, article 27849, 2016.

[56] K. Miura, Y. Kodama, S. Inokuchi et al., “Toll-like receptor 9 promotes steatohepatitis by induction of interleukin- $1 \beta$ in mice," Gastroenterology, vol. 139, no. 1, pp. 323-334.e7, 2010.

[57] J. Petrasek, A. Dolganiuc, T. Csak, E. A. Kurt-Jones, and G. Szabo, "Type I interferons protect from Toll-like receptor 9-associated liver injury and regulate IL-1 receptor antagonist in mice," Gastroenterology, vol. 140, no. 2, pp. 697-708.e4, 2011.

[58] R. Medzhitov, "Toll-like receptors and innate immunity," Nature Reviews Immunology, vol. 1, no. 2, pp. 135-145, 2001.

[59] T. Duparc, H. Plovier, V. G. Marrachelli et al., "Hepatocyte MyD88 affects bile acids, gut microbiota and metabolome contributing to regulate glucose and lipid metabolism," Gut, vol. 66, no. 4, pp. 620-632, 2017. 
[60] K. Miura, E. Seki, H. Ohnishi, and D. A. Brenner, "Role of tolllike receptors and their downstream molecules in the development of nonalcoholic Fatty liver disease," Gastroenterology Research and Practice, vol. 2010, Article ID 362847, 9 pages, 2010.

[61] T. Hosoi, S. Yokoyama, S. Matsuo, S. Akira, and K. Ozawa, "Myeloid differentiation factor 88 (MyD88)-deficiency increases risk of diabetes in mice," PLoS One, vol. 5, no. 9, p. e12537, 2010

[62] A. Everard, L. Geurts, R. Caesar et al., "Intestinal epithelial MyD88 is a sensor switching host metabolism towards obesity according to nutritional status," Nature Communications, vol. 5, article 5648, 2014.

[63] F. S. Sutterwala, Y. Ogura, and R. A. Flavell, "The inflammasome in pathogen recognition and inflammation," Journal of Leukocyte Biology, vol. 82, no. 2, pp. 259-264, 2007.

[64] J. Henao-Mejia, E. Elinav, C. Jin et al., "Inflammasome-mediated dysbiosis regulates progression of NAFLD and obesity," Nature, vol. 482, no. 7384, pp. 179-185, 2012.

[65] L. G. Albenberg and G. D. Wu, "Diet and the intestinal microbiome: associations, functions, and implications for health and disease," Gastroenterology, vol. 146, no. 6, pp. 1564-1572, 2014.

[66] S. Bibbo, G. Ianiro, V. Giorgio et al., "The role of diet on gut microbiota composition," European Review for Medical and Pharmacological Sciences, vol. 20, no. 22, pp. 4742-4749, 2016.

[67] F. Laugerette, C. Vors, A. Géloën et al., "Emulsified lipids increase endotoxemia: possible role in early postprandial low-grade inflammation," The Journal of Nutritional Biochemistry, vol. 22, no. 1, pp. 53-59, 2011.

[68] F. J. Gonzalez, C. Jiang, and A. D. Patterson, “An intestinal microbiota-farnesoid X receptor axis modulates metabolic disease," Gastroenterology, vol. 151, no. 5, pp. 845-859, 2016.

[69] N. Kobyliak, C. Conte, G. Cammarota et al., "Probiotics in prevention and treatment of obesity: a critical view," Nutrition \& Metabolism, vol. 13, no. 1, p. 14, 2016.

[70] F. Mofidi, H. Poustchi, Z. Yari et al., "Synbiotic supplementation in lean patients with non-alcoholic fatty liver disease: a pilot, randomised, double-blind, placebo-controlled, clinical trial," British Journal of Nutrition, vol. 117, no. 05, pp. 662668, 2017. 




The Scientific World Journal
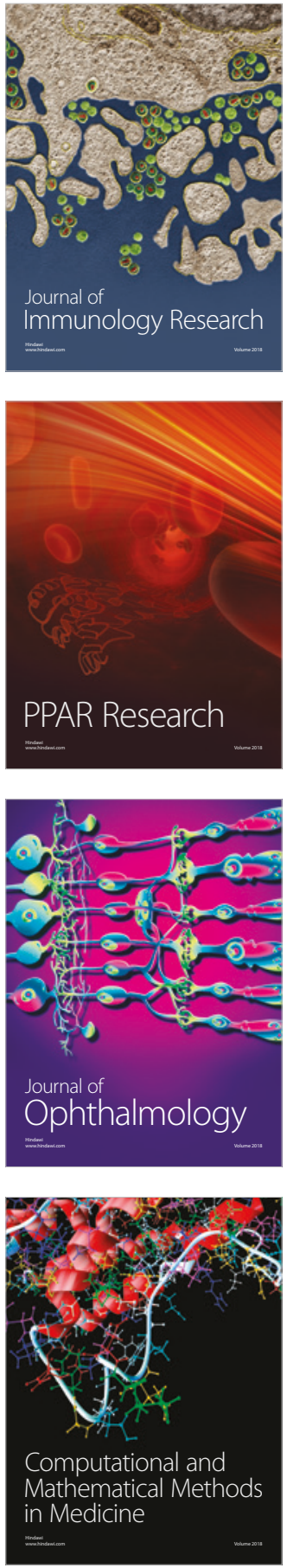

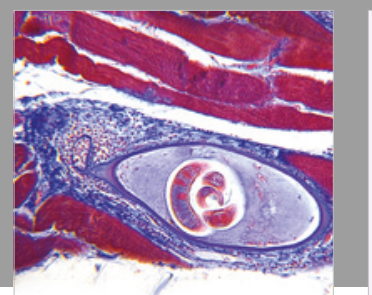

Gastroenterology Research and Practice

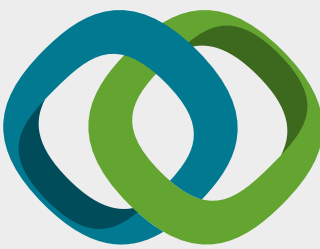

\section{Hindawi}

Submit your manuscripts at

www.hindawi.com
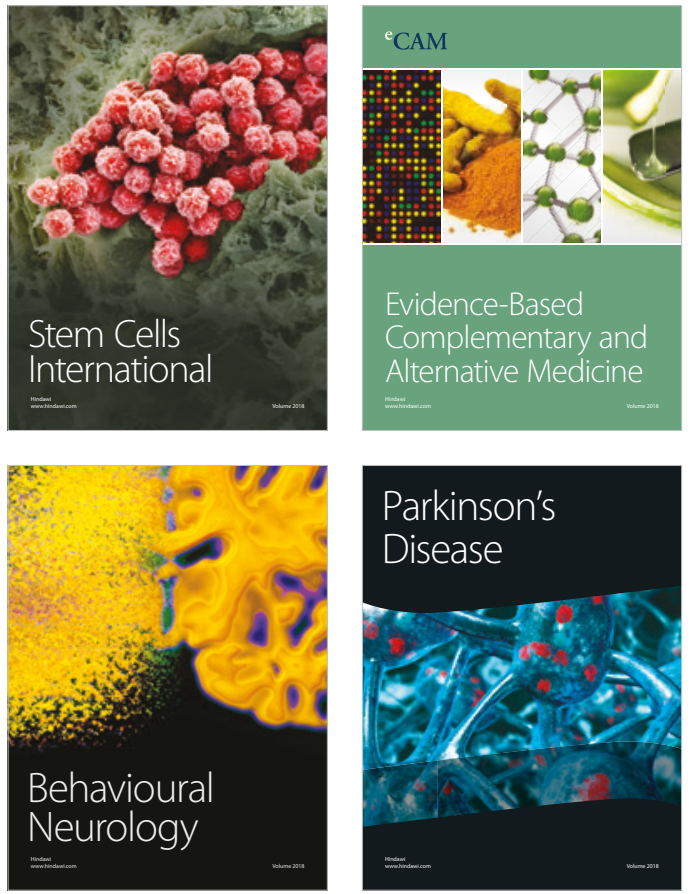

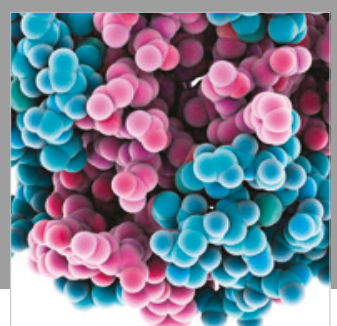

ournal of

Diabetes Research

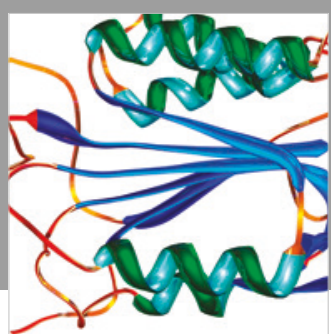

Disease Markers
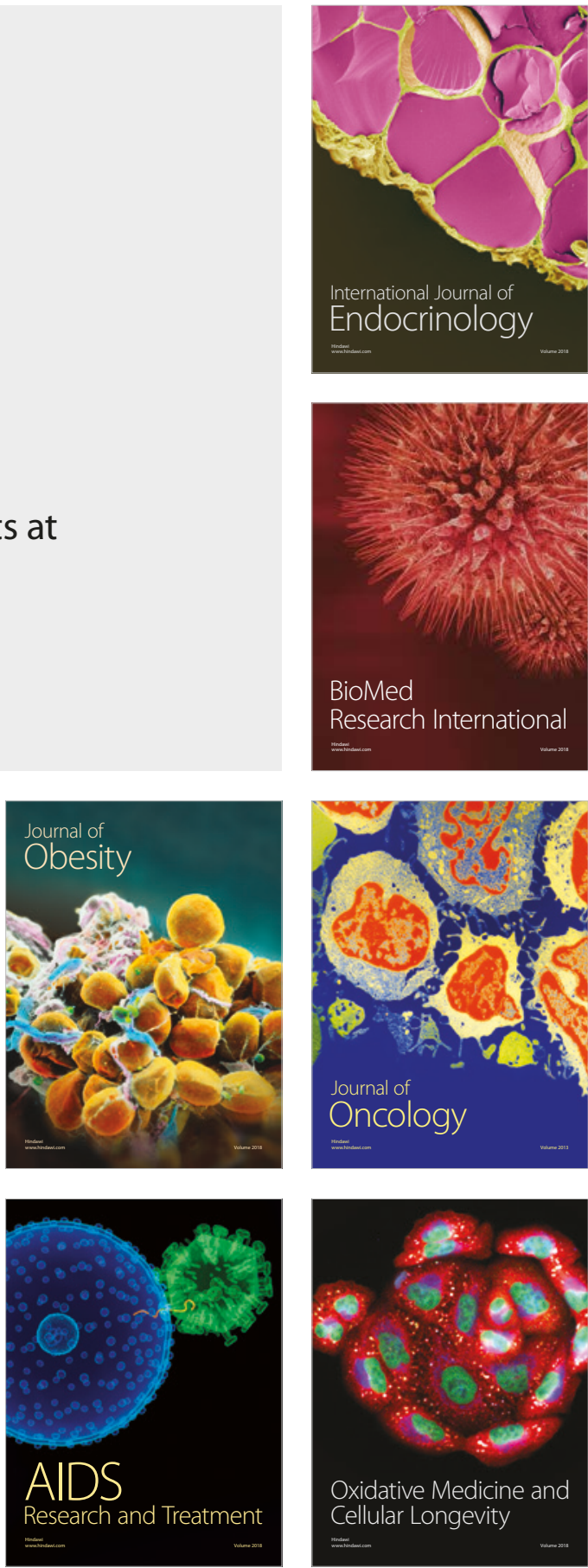